CERN-TH/2000-352

Bicocca-FT-00-23

\title{
Branes from Unstable Systems of Branes
}

\author{
Laurent Houart \\ Dipartimento di Fisica \\ Università Milano Bicocca \\ Piazza delle Scienze 3, Milano, Italy \\ Laurent.Houart@mi . infn. it \\ and \\ Yolanda Lozano \\ Theory Division, CERN \\ 1211 Genève 23, Switzerland \\ yolanda.lozano@cern.ch
}

\begin{abstract}
We discuss various aspects of the description of branes as topological solitons in unstable brane systems of higher dimensions. We first describe a classification of all the possible realisations of branes of $\mathrm{M}$ and type II theories as topological solitons of a brane-antibrane system. We then present a description of type IIB NS-NS $p$-branes in terms of topological solitons in systems of spacetime-filling NS9, anti-NS9 pairs and discuss the implications of these constructions in the description of BPS and non-BPS states in the strongly coupled Heterotic $\mathrm{SO}(32)$ theory. We finally present briefly the construction of a conjectured spacetime-filling non-BPS M10-brane, starting point for a brane descent construction of the branes of M-theory.
\end{abstract}

\footnotetext{
${ }^{1}$ To be published in the proceedings of the RTN-meeting The Quantum Structure of Spacetime and the Geometric Nature of Fundamental Interactions, Berlin, October 2000.
} 


\section{Introduction and Summary}

Recently, significant progress has been made in our understanding of Mtheory beyond the BPS configurations. These advances have already permitted to test the web of dualities relating the different phases of M-theory (the different superstring theories) on some of the non-BPS states of the spectrum. A beautiful outlook on the interplays between BPS branes and non-BPS branes has also been given in some cases (see [1] for reviews and references therein) along with an elegant mathematical formulation [2, 3, 4]. For example, a BPS Dp-brane in type II may be viewed as coming from a non-BPS system given by a $\mathrm{D}(\mathrm{p}+2)$, anti- $\mathrm{D}(\mathrm{p}+2)$ pair. The instability of this non-BPS configuration manifests itself in a complex tachyonic mode of the open string stretched between the pair. When the pair coincides, the tachyon can roll down to a true vacuum and condenses in a topologically non-trivial way leading to a stable vortex-like configuration, and the resulting object is a BPS Dp-brane [5], [6].

Most of the progress has been made in the context of D-branes. It would certainly be interesting to understand if similar constructions exist for the other $p$-branes like for instance the M-branes or the NS-NS charged ones. We will describe here some aspects of this problematic. We will first discuss a systematic classification of all the possible realisations of branes of $\mathrm{M}$ and type II theories as topological solitons of brane-antibrane systems. This classification of all the possibilities, consistent with the structure of the theory, is achieved by studying the Wess-Zumino terms in the worldvolume effective actions of the branes of M-theory and their reductions. We will illustrate the method on the MKK- $\overline{\mathrm{MKK}}$ system. We will then turn to a description of the brane descent construction in type IIB starting with a spacetime-filling brane-antibrane system NS-NS charged and discuss the implications of this construction in the description of BPS and non-BPS states in the strongly coupled heterotic SO(32) theory. Finally we will construct the worldvolume action of a conjectured spacetime-filling non-BPS M10-brane, starting point for a brane descent construction in M-theory. This talk is based on [7], [8] and [9] where further details can be found.

\section{Realisation of branes as topological solitons}

The (bosonic) low energy effective worldvolume theory of a Dp, anti-Dp pair is described by two $\mathrm{U}(1)$ field strengths, one $F=d A$ for the brane and one $F^{\prime}=d A^{\prime}$ for the antibrane, along with a complex tachyon field charged under the relative $\mathrm{U}(1)$. The $\mathrm{D}(\mathrm{p}-2)$ brane is obtained as a topological soliton of 
the system after the tachyon condensates, rolling down to its true vacuum in a topological non-trivial way, giving rise to a usual vortex solution [6], [5]. The fact that this codimension two object carries a $\mathrm{D}(\mathrm{p}-2)$ brane charge can be deduced from the following Wess-Zumino term in the action of the pair:

$$
\int_{\Sigma_{p+1}} C^{(p-1)} \wedge\left(F-F^{\prime}\right)
$$

which indicates, after integration on the codimensions, that the vortex is charged under the RR-field $C^{(p-1)}$ and corresponds thus to a $\mathrm{D}(\mathrm{p}-2)$ brane.

Having understood the construction for Dp-branes and using the Web of dualities relating the different phases of M-theory, it is possible to qualitatively discuss all the possible realisations of branes of $\mathrm{M}$ and type II theories as topological solitons in brane-antibrane systems. The classification of all the possibilities is achieved by studying the Wess-Zumino terms in the worldvolume effective actions of the branes of M-theory and their reductions. We will here illustrate the method by analysing the Kaluza-Klein, anti KaluzaKlein monopole pair (MKK- $\overline{\mathrm{MKK}}$ ) and refer the reader to [7] for the other cases and the complete classification.

The worldvolume effective action of the M-theory Kaluza-Klein monopole was constructed in [10, 11]. The existence of the Taub-NUT direction in the space transverse to the monopole is implemented at the level of the effective action by introducing a Killing isometry which is gauged in the worldvolume. Then the target space fields must couple in the worldvolume with covariant derivatives of the embedding scalars, or through contraction with the Killing vector. The Kaluza-Klein monopole is charged with respect to an 8-form, which is the electric-magnetic dual of the Killing vector considered as a 1form. This field is itself contracted with the Killing vector, giving a 7-form minimally coupled to the 7 dimensional worldvolume of the monopole.

The worldvolume effective action of the monopole contains the following term [11]:

$$
\int_{R^{6+1}} i_{\hat{k}} \hat{\tilde{C}} \wedge d \hat{b}^{(1)}
$$

where $\hat{\tilde{C}}$ denotes the 6 -form of eleven dimensional supergravity, $\hat{k}$ is the Killing vector, with $\left(i_{\hat{k}} \hat{\tilde{C}}\right)_{\hat{\mu}_{1} \ldots \hat{\mu}_{5}} \equiv \hat{k}^{\hat{\mu}_{6}} \hat{\tilde{C}}_{\hat{\mu}_{1} \ldots \hat{\mu}_{6}}$, and $\hat{b}^{(1)}$ is a 1 -form worldvolume field which describes the coupling to an M2-brane wrapped on the Taub-NUT direction. The same coupling appears in the effective action of the MKK- $\overline{\text { MKK }}$ pair, where now $\hat{b}^{(1)}=\hat{b}_{1}^{(1)}-\hat{b}_{2}^{(1)}$, with $\hat{b}_{1,2}^{(1)}$ the corresponding vector field in the worldvolume of each monopole. This term allows for an object of codimension 2, associated to the localised magnetic flux which accompanies the topologically non-trivial condensation of the tachyonic mode 
of the M2-brane. The integration of the localised flux $d \hat{b}^{(1)}$ on the codimensions gives

$$
\int_{R^{4+1}} i_{\hat{k}} \hat{\tilde{C}}
$$

i.e. the solution is an M5-brane soliton with one worldvolume direction wrapped around the Taub-NUT direction. Therefore we can describe a (wrapped) M5-brane soliton as a bound state of a pair of Kaluza-Klein anti Kaluza-Klein monopoles.

We can now analyse the different possible configurations in the type IIA theory to which this process gives rise. Dimensionally reducing along the Taub-NUT direction of the monopoles we can describe a D4-brane through the condensation of the tachyonic mode of an open string between a D6, anti-D6 pair [6]. This is described by the coupling:

$$
\int_{R^{6+1}} C^{(5)} \wedge d b^{(1)}
$$

which is straightforwardly obtained by reducing the coupling (2.2) describing the creation of the solitonic (wrapped) M5-brane.

The reduction along a worldvolume direction of the monopoles gives as one of the possible configurations a solitonic NS5-brane, obtained after the condensation of the tachyonic excitation of an open string stretched between a Type IIA pair of Kaluza-Klein anti-Kaluza-Klein monopoles. The worldvolume reduction of (2.2) gives indeed:

$$
\int_{R^{5+1}} i_{k} C^{(5)} \wedge d b^{(1)}+\int_{R^{5+1}} i_{k} B^{(6)} \wedge d b^{(0)}
$$

where $b^{(0)}$ arises as the component of $\hat{b}^{(1)}$ along the worldvolume direction that is being reduced. These terms describe two processes: One in which a (wrapped) D4-brane is created after condensation of the tachyonic mode of a (wrapped) D2-brane stretched between two Type IIA monopoles, described by the first term, and one in which a (wrapped) NS5-brane is created after the condensation of a (wrapped) open string. This is, to our knowledge, the first example in which a NS5-brane has been described through a brane anti-brane pair annihilation.

We now turn to the "dual" process in the MKK- $\overline{\mathrm{MKK}}$ system, i.e. obtaining an M2-brane soliton after the condensation of an M5-brane. A wrapped M5-brane is coupled in the worldvolume of the MKK through a 4 -form field, which is the worldvolume dual of the vector field describing the coupling of a wrapped M2-brane.

The Wess-Zumino term of the dual description of the MKK- $\overline{\mathrm{MKK}}$ system was found in [7], and it is given by: 


$$
\int_{R^{6+1}}\left(i_{\hat{k}} \hat{C}\right) \wedge d \hat{b}^{(4)}
$$

where $d \hat{b}^{(4)}$ is the worldvolume dual of the center of mass vector field $\hat{b}_{1}^{(1)}+\hat{b}_{2}^{(1)}$. This term allows for a codimension 5 soliton corresponding to a wrapped M2brane. Indeed, after integrating the localised $d \hat{b}^{(4)}$ flux on the codimensions one obtains:

$$
\int_{R^{1+1}}\left(i_{\hat{k}} \hat{C}\right)
$$

which is the charge of a wrapped M2-brane.

The reduction along the Taub-NUT direction of the coupling (2.6) gives:

$$
\int_{R^{6+1}} B^{(2)} \wedge d b^{(4)}
$$

Therefore, we find after the integration of the 5 -form field strength a solitonic object of codimension 5 charged under $B^{(2)}$, namely a fundamental string, obtained after the condensation of a streched D4 between a D6, anti-D6 pair. Processes giving rise to fundamental strings where first study in [12].

Two other possible brane anti-brane annihilation processes can be deduced in Type IIA from the M-theory Kaluza-Klein anti-Kaluza-Klein annihilation that we have just discussed. If we reduce this process along a worldvolume direction of the Kaluza-Klein monopoles we find two terms. The first term describes a fundamental string created after the condensation of a NS5-brane stretched between a pair of Type IIA Kaluza-Klein monopole anti-monopole. Both the fundamental string and the NS5-brane are wrapped on the Taub-NUT direction of the monopole. On the other hand, the second term represents a wrapped D2-brane arising after the condensation of a wrapped D4-brane stretched between the two monopoles.

\section{Spacetime-filling NS-NS branes and brane descent relations}

In this section, we first discuss the possibility of describing the NS-NS charged branes of type IIB in terms of a system of spacetime-filling NS9, anti-NS9 pairs [8].

The NS9 is predicted by the spacetime SUSY algebra of the type IIB theory, and its worldvolume effective action was constructed in [13]. The explicit form of the Wess-Zumino part describing $N$ NS9-branes reads: 


$$
\begin{aligned}
& S_{\mathrm{NS} 9}^{\mathrm{WZ}}=\int_{R^{9+1}} \operatorname{Tr}\left[B^{(10)}+\tilde{C}^{(8)} \wedge \tilde{F}+B^{(6)} \wedge \tilde{F} \wedge \tilde{F}+C^{(4)} \wedge \tilde{F} \wedge \tilde{F} \wedge \tilde{F}+\right. \\
& \left.+B^{(2)} \wedge \tilde{F} \wedge \tilde{F} \wedge \tilde{F} \wedge \tilde{F}+\frac{C^{(0)}}{\left(C^{(0)}\right)^{2}+e^{-2 \phi}} \tilde{F} \wedge \tilde{F} \wedge \tilde{F} \wedge \tilde{F} \wedge \tilde{F}\right]
\end{aligned}
$$

Here $\tilde{F} \in U(N)$ describes D1-branes ending on the NS9-branes, $B^{(10)}$ is the NS-NS 10-form potential with respect to which the NS9-brane is charged and $\tilde{C}^{(8)}$ is the S-dual of the 8-form RR-potential (see [14]).

The effective action describing NS9, anti-NS9 pairs contains additional worldvolume fields. One has, in addition to $\tilde{F}$, a second field strength $\tilde{F}^{\prime}$, associated with the antibranes, and a complex charged tachyon field $T$. However, to discuss the possible realisations of p-branes as topological defects of the NS9, anti-NS9 system one can focalise on the WZ terms of the brane action (3.1), bearing in mind that the topologically non-trivial character of the soliton can be carried by one of the two field strengths, say $\tilde{F}$ (see for instance [6]).

The analysis of the NS9-brane WZ action shows that the NS-NS branes of the type IIB theory can be understood as bound states of NS9, anti-NS9 pairs of branes. The different possible classical solutions corresponding to topological defects of codimension $2 k$ can be obtained starting with $N=2^{k-1}$ pairs of NS9, anti-NS9 using the Atiyah Bott Shapiro realisation [15] of the generator of the homotopy group $\pi_{2 k-1}\left(\left(U\left(2^{k-1}\right)\right)\right.$, as explained in [2] for the case of the D9, anti-D9 system. The general pattern that is derived from this analysis is: D7 $=(\mathrm{NS} 9$, anti-NS9), NS5 $=2(\mathrm{NS} 9$, anti-NS9), D3=4 (NS9, anti-NS9), $\mathrm{F} 1=8(\mathrm{NS} 9$, anti-NS9) and $\mathrm{D}(-1)=16$ (NS9, anti-NS9), of type IIB p-branes as bound states of NS9, anti-NS9 pairs. Therefore, we see that all the branes predicted by the analysis of the type IIB spacetime supersymmetry algebra, apart from the pp-wave and the Kaluza-Klein monopole, can be realised as bound states of any of the two types of spacetime-filling branes of the theory. Of course for this to hold the NS9-branes must be considered on an equal footing with the RR 9-branes. The pp-wave and the KaluzaKlein monopole solutions are only defined in spacetimes with one special, isometric, direction. In the case of the pp-wave this is the direction of propagation of the wave, whereas in the monopole case this is the Taub-NUT fiber of the transverse space. Therefore these branes cannot be understood as bound states of spacetime-filling branes that do not see any of these special directions.

We know turn to the implications of these constructions in the description of BPS and non-BPS states in the strongly coupled Heterotic SO(32) theory. 
It was argued in [16] [13] that the Heterotic string with gauge group $\mathrm{SO}(32)$ could be obtained as a nonperturbative orientifold construction of the type IIB theory. This construction is determined by S-duality: If the type I theory can be defined as an orientifold of the type IIB theory by its worldsheet parity reversal symmetry, its S-dual, i.e. the Heterotic SO(32), should be defined at strong coupling as an orientifold of the type IIB theory by the S-dual operation of worldsheet parity reversal. Perturbatively, this operation coincides with the $(-1)^{F_{L}}$ symmetry of the type IIB theory, where $F_{L}$ is the left-moving fermion number. Modding out the type IIB theory by $(-1)^{F_{L}}$ gives rise to the type IIA string, and this is determined by the twisted sector that has to be added in order to restore modular invariance. However one can consider adding anomaly cancelling 9-branes as in the type I theory, and this is the way in which the Heterotic string can be produced. S-duality determines that the background 9-branes must be NS9-branes, and also that, nonperturbatively, $(-1)^{F_{L}}$ can be defined as the operation reversing the orientation of a D-string. This worldsheet operation has associated an orientifold fixed plane with -32 NS-NS charge, and this charge is cancelled through the addition of 32 NS9-branes, with one unit of NS-NS charge. Together they reproduce the gauge sector of the Heterotic SO(32) supergravity 13.

The massless spectrum of the Heterotic F-string can be described in terms of open D-strings with both ends on the F1, DD D-strings, and with one end on the F1 and the other on an NS9-brane, DN D-strings, [13. The DD Dstrings contribute with 8 scalars and 8 right-handed Majorana-Weyl spinors, whereas the DN D-strings contribute with 32 left-moving fermions. This description of the Heterotic string arises at strong coupling. However, given that the massless states are BPS, it can be as well extrapolated to the weak coupling regime. In this limit the $\mathrm{SO}(32)$ charges tethered to the $\mathrm{F} 1$ are pulled onto its worldsheet, since $\tau_{\mathrm{D} 1} / \tau_{\mathrm{F} 1} \sim 1 / g_{s} \rightarrow \infty$ and the D-strings collapse to a point, giving the usual $\mathrm{SO}(32)$ Heterotic worldsheet currents.

So far the discussion has been focussed on the massless BPS states. The Heterotic $\mathrm{SO}(32)$ theory contains as well perturbative massive states in the spinorial representation of $\mathrm{SO}(32)$, which are non-BPS but stable, given that they are the lightest states transforming as spinors of $\mathrm{SO}(32)$. Sen [6], [5] showed that the correct way of describing these states at strong coupling is in terms of a weakly coupled type I (D1, anti-D1) system. A (D1, anti-D1) pair is unstable due to the presence of a tachyonic mode in the open strings stretched between the two branes, and moreover, since the two branes are spinors under $\mathrm{SO}(32)$ the bound state cannot transform as a spinor. However, compactifying the D-strings and switching on a $Z_{2}$ Wilson line the tachyonic mode can condense into a stable configuration different from the vacuum, 
and transforming as a spinor of $\mathrm{SO}(32)$ [6].

We can now take the following point of view to describe the spinorial non-BPS states of the Heterotic theory in the strong coupling regime. A bound state $\mathrm{D} 0=(\mathrm{D} 1$, anti-D1) in the weakly coupled type I side predicts a bound state (F1, anti-F1) in the strongly coupled Heterotic theory. S-duality determines that each of these F1's transforms in the spinor representation of $\mathrm{SO}(32)$, since DN open D-strings contribute with these quantum numbers, and that the whole system is unstable due to the presence of a tachyonic mode in the open D-strings stretched between the two F1's. S-duality determines as well that if the system is compactified a state transforming as a spinor under $\mathrm{SO}(32)$ should emerge after the condensation of the tachyonic mode. This description at strong coupling in terms of an (F1, anti-F1) bound state is also natural from another point of view. In the Heterotic theory the non-BPS states in the spinorial representation of $\mathrm{SO}(32)$ correspond to unwrapped fundamental strings, i.e. to strings not charged with respect to the NS-NS 2 -form potential. This charge cancellation is simply achieved by the F1, anti-F1 superposition.

It is also interesting to point out that the Heterotic fundamental string can arise as a bound state of a pair of NS5, anti-NS5 branes, since this is the S-dual configuration of the D1=(D5, anti-D5) bound state in type I $6 \|$. This is implied by S-duality, and can be read as well directly from the NS5-brane effective action truncated to a Heterotic background, in particular from the term [17]: $\int_{R^{5+1}} \operatorname{Tr}\left[B^{(2)} \wedge \tilde{F} \wedge \tilde{F}\right]$. In turn, the NS5-brane is obtained as a bound state of four NS9, anti-NS9 pairs of branes. This is S-dual to the D5 $=4(\mathrm{D} 9$, anti-D9) configuration in type I, and can also be derived from the NS9-brane effective action (3.1) truncated to a Heterotic background. Indeed, by S-duality, four NS9, anti-NS9 are characterised by an $\mathrm{SO}(4) \times$ $\mathrm{SO}(4)$ gauge symmetry. One of the $\mathrm{SU}(2)$ of the $\mathrm{SO}(4)$ Chan-Paton group, characterising, say, the four anti-NS9 branes, is used in the construction of the NS5-brane as an instanton configuration and the other $\mathrm{SU}(2)$ survives as its gauge symmetry. The Heterotic 5-brane contains as well SO(32) currents that arise from open D-strings with DN boundary conditions, i.e. with one end on an NS9-brane [16]. Therefore, each brane of the NS5, anti-NS5 pair has an $\mathrm{SU}(2) \times \mathrm{SO}(32)$ gauge structure, from which the $\mathrm{SU}(2)$ is used in the instanton construction of the Heterotic F-string and the $\mathrm{SO}(32)$ group survives as its gauge structure. 


\section{A non-BPS M10-brane}

In this section we briefly discuss how to construct a non-BPS M10-brane 9]. The conjectured M10-brane is an unstable spacetime-filling brane in massive eleven dimensional supergravity. This brane is constructed such that the BPS M9-brane is obtained after the tachyonic mode of an M2-brane ending on it condenses and such that it gives the non-BPS D9-brane of type IIA upon reduction.

The study of 9-branes and 10-branes in M-theory should be carried out in the context of massive eleven dimensional supergravity, given that the BPS M9-brane couples magnetically to the mass. It is well-known that a fully eleven dimensional Lorentz invariant massive supergravity cannot be constructed [18]. Nevertheless, the massive Type IIA supergravity of Romans [19] can be derived from eleven dimensions if the condition of eleven dimensional Lorentz invariance is relaxed and one assumes an isometric eleventh direction that is gauged in the supergravity action [20]. Accordingly, the branes living in this massive eleven dimensional background are described by worldvolume effective actions where the Killing direction, characterised by a Killing vector $\hat{k}$, is gauged. The M9-brane effective action has been constructed in [21], where it is shown that its worldvolume field strength is a two-form $\hat{\mathcal{F}}$ characterising an M2, wrapped on the Killing direction, ending on the M9. The worldvolume theory of the M10 will also contain this $\hat{\mathcal{F}}$ field but will contain in addition a real tachyon field $\hat{T}$ such that upon double dimensional reduction the type IIA non-BPS D9-brane [3] is reproduced. Under the two hypothesis made, one can thus construct the action of a non-BPS M10-brane. Here we just present the result. The action is: $S^{(\mathrm{M} 10)}=S_{\mathrm{DBI}}^{(\mathrm{M} 10)}+S_{\mathrm{WZ}}^{(\mathrm{M} 10)}$, where the DBI part is given by:

$$
S_{\mathrm{DBI}}^{(\mathrm{M} 10)}=-\int_{R^{9+1}}|\hat{k}|^{3} \sqrt{\left|\operatorname{det}\left(\hat{\Pi}+|\hat{k}|^{-1} \hat{\mathcal{F}}\right)\right|} \hat{R}(\hat{T}, \partial \hat{T}, \ldots) .
$$

Here $\hat{\Pi}$ is the pull-back of the spacetime metric:

$$
\hat{\Pi}=D \hat{X}^{\hat{\mu}} D \hat{X}^{\hat{\nu}} \hat{g}_{\hat{\mu} \hat{\nu}}=\partial \hat{X}^{\hat{\mu}} \partial \hat{X}^{\hat{\nu}}\left(\hat{g}_{\hat{\mu} \hat{\nu}}+|\hat{k}|^{-2} \hat{k}_{\hat{\mu}} \hat{k}_{\hat{\nu}}\right),
$$

and $\hat{R}$ is some function of the tachyon field vanishing at the minimum of the tachyon potential, where the action then vanishes identically and describes a configuration indistinguishable from the vacuum. For vanishing tachyon field $\hat{R}$ gives a constant. For a tachyonic kink configuration in the zero size limit $\hat{R}(x) \sim C \delta\left(x-x_{0}\right)$, and (4.1) reduces to the DBI part of the effective action of a BPS M9-brane. The Wess-Zumino part is given by the following expression: 


$$
\begin{aligned}
& S_{\mathrm{WZ}}^{(M 10)}=\int_{R^{9+1}}\left[i_{\hat{k}} \hat{B}^{(10)}+i_{\hat{k}} \hat{N}^{(8)} \wedge \hat{\mathcal{F}}+i_{\hat{k}} \hat{\tilde{C}} \wedge \hat{\mathcal{F}} \wedge \hat{\mathcal{F}}+\hat{C}_{\hat{\mu}_{1} \hat{\mu}_{2} \hat{\mu}_{3}} .\right. \\
& \left.. D \hat{X}^{\hat{\mu}_{1}} D \hat{X}^{\hat{\mu}_{2}} D \hat{X}^{\hat{\mu}_{3}} \wedge \hat{\mathcal{F}} \wedge \hat{\mathcal{F}} \wedge \hat{\mathcal{F}}+\hat{A} \wedge \hat{\mathcal{F}} \wedge \hat{\mathcal{F}} \wedge \hat{\mathcal{F}} \wedge \hat{\mathcal{F}}+\ldots\right] \wedge d \hat{T},
\end{aligned}
$$

where $\hat{B}^{(10)}$ is the spacetime field electric-magnetic dual to the mass and $\hat{N}^{(8)}$ is the Kaluza-Klein monopole charge.

Having this spacetime-filling M10-brane it is possible to describe brane descent constructions of all M-branes. We refer the interested reader to [9].

\section{Acknowledgements}

L. H. would like to acknowledge the support of the European Commission RTN programme RTN1-1999-00116.

\section{References}

[1] A. Sen, Non-BPS States and Branes in String Theory, hep-th/9904207; A. Lerda and R. Russo, Stable Non-BPS States in String Theory: a Pedagogical Review, hep-th/9905006; J. H. Schwarz, TASI Lectures on Non-BPS D-Brane Systems, hep-th/9908144; O. Bergman and M. Gaberdiel, NonBPS Dirichlet Branes, hep-th/9908126.

[2] E. Witten, D-Branes and K-Theory, J. High Energy Phys. 9812 (1998) 019, hep-th/9810188.

[3] P. Hor̆ava, Type IIA D-Branes, K-Theory, and Matrix Theory, Adv. Theor. Math. Phys. 2 (1999) 1373, hep-th/9812135.

[4] O. Bergman, E. Gimon and P. Hor̆ava, Brane Transfer Operations and T-Duality of Non-BPS States, J. High Energy Phys. 9904 (1999) 010, hep-th/9902160.

[5] A. Sen, Tachyon Condensation on the Brane-Antibrane System, J. High Energy Phys. 9808 (1998) 012, hep-th/9805170.

[6] A. Sen, SO(32) Spinors of Type $I$ and Other Solitons on Brane -Antibrane Pair, J. High Energy Phys. 9809 (1998) 023, hep-th/9808141.

[7] L. Houart and Y. Lozano, Type II Branes from Brane-Antibrane in Mtheory, Nucl. Phys. B575 (2000) 195, hep-th/9910266. 
[8] L. Houart and Y. Lozano, S-Duality and Brane Descent Relations, JHEP 003 (2000) 031, hep-th/9911173.

[9] L. Houart and Y. Lozano, Brane Descent Relations in M-theory, Phys. Lett. B479 (2000) 299, hep-th/0001170.

[10] E. Bergshoeff, B. Janssen and T. Ortín, Kaluza-Klein Monopoles and Gauged Sigma-Models, Phys. Lett. B410 (1997) 132, hep-th/9706117.

[11] E. Bergshoeff, E. Eyras and Y. Lozano, The Massive Kaluza-Klein Monopole, Phys. Lett. B430 (1998) 7r, hep-th/9802199.

[12] P. Yi, Membranes from Five-Branes and Fundamental Strings from Dp Branes, Nucl. Phys. B550 (1999) 214, hep-th/9901159.

[13] E. Bergshoeff, E. Eyras, R. Halbersma, C.M. Hull, Y. Lozano and J.P. van der Schaar, Spacetime-filling Branes and Strings with Sixteen Supercharges, Nucl. Phys. B564 (2000) 29 , hep-th/9812224.

[14] E. Eyras and Y. Lozano, Exotic Branes and Nonperturbative Seven Branes, Nucl. Phys. B573 (2000) 735, hep-th/9908094.

[15] M. F. Atiyah, R. Bott and A. Shapiro, Clifford Modules, Topology 3 (1964) 3.

[16] C.M. Hull, The Non-perturbative SO(32) Heterotic String, Phys. Lett. B462 (1999) 271, hep-th/9812210.

[17] E. Eyras, B. Janssen and Y. Lozano, 5-branes, KK-monopoles and Tduality, Nucl. Phys. B531 (1998) 275, hep-th/9806169.

[18] K. Bautier, S. Deser, M. Henneaux and D. Seminara, No Cosmological D=11 Supergravity, Phys. Lett. B406 (1997) 49, hep-th/9704131.

[19] L.J. Romans, Massive $N=2 a$ Supergravity in Ten Dimensions, Phys. Lett. B169 (1986) 374.

[20] E. Bergshoeff, Y. Lozano and T. Ortín, Massive Branes, Nucl. Phys. B518 (1998) 363, hep-th/9712115.

[21] E. Bergshoeff and J.P. van der Schaar, On M9-branes, Class. Quant. Grav. 16 (1999) 23, hep-th/9806069; E. Eyras and Y. Lozano, Brane Actions and String Dualities, hep-th/9812225; T. Sato, A 10-form Gauge Potential and an M-9-brane Wess-Zumino Action in Massive 11D Theory, Phys. Lett. B477 (2000) 45\%, hep-th/9912030. 